Proceedings of the 17th Czech and Slovak Conference on Magnetism, Košice, Slovakia, June 3-7, 2019

\title{
Effect of Temperature on Magnetization Curves near Curie Point in LaFeCoSi Alloy
}

\author{
R. Gozdur ${ }^{a}$, P. GęBARA ${ }^{b, *}$ And K. ChWASteK ${ }^{c}$ \\ ${ }^{a}$ Łódź University of Technology, Department of Semiconductor and Optoelectronics Devices, \\ Wólczańska 211/215, 90-924 Łódź, Poland \\ ${ }^{b}$ Institute of Physics, Czestochowa University of Technology, al. Armii Krajowej 19, 42-200 Czestochowa, Poland \\ ${ }^{c}$ Faculty of Electrical Engineering, Częstochowa University of Technology, \\ al. Armii Krajowej 17, 42-200 Częstochowa, Poland
}

\begin{abstract}
Analytical description of the effect of temperature on magnetization curves can be of interest to practitioners working on magnetocaloric effect. This paper compares two models, derived from the phenomenological $T(x)$ model. The hybrid product-T(x) model offers an improved description of magnetization for temperatures close to the Curie point, as compared with the original model with the linear reversibility term, which is verified using experimental data for $\mathrm{La}(\mathrm{Fe}, \mathrm{Co}, \mathrm{Si})_{13}$ magnetocaloric alloy.
\end{abstract}

DOI: 10.12693/APhysPolA.137.918

PACS/topics: 75.30.Sg, 75.50.Bb

\section{Introduction}

The magnetocaloric effect (MCE) relies on changing temperature of a magnetocaloric material (MCM) subjected to magnetization in adiabatic conditions. The MCE is known for over a century [1], however, over the years it used to be the subject of study for a relatively narrow group of researchers. An enormous interest of scientists in the MCE and its potential applications began in 1997, when the giant MCE was discovered in the $\mathrm{Gd}_{5}\left(\mathrm{Si}_{2} \mathrm{Ge}_{2}\right)$ compound $[2,3]$. Since then, the number of papers on the MCE has started to grow up exponentially and it is at present one of the "hottest" topics in magnetism. This is related to the possible use of the MCE in magnetic cooling [4-8]. The $\mathrm{La}(\mathrm{Fe}, \mathrm{Si})_{13}$-based alloys are an important group of MCMs, as they are relatively cheap and the MCE occurs in these materials in the near-room temperature [9]. Moreover, tuning of alloy properties is realized by partial substitution of $\mathrm{La}$ atoms with other lanthanides [10-12] or by replacing the Fe atoms with transition metals [13-15]. Such modifications of chemical composition affect significantly the Curie point $T_{\mathrm{C}}$ and the change in magnetic entropy $\Delta S_{M}$. Most of the papers on $\mathrm{La}(\mathrm{Fe}, \mathrm{Si})_{13}$-based alloys focus on their processing methods, microstructure, and magnetic properties. From the scientific point of view, it is also important to investigate various effects in the vicinity of phase transition (PT) point observed for the material. The response of magnetic material near the $\mathrm{PT}$ point can be studied by applying an interesting method of modeling the hysteresis curves [16]. The present paper focuses on the possibility to describe the $M-H$ curves near the

*corresponding author; e-mail: pgebara@wip.pcz.pl
$T_{\mathrm{C}}$ with the use of the $T(x)$ model [17] and its modification [18]. So far, either the Preisach or the JA models have been used for the description of magnetization curves in MCMs [19, 20]. The favorable features of $T(x)$ derived descriptions in comparison to other commonly used models are rigorous mathematical foundations and simplicity of use.

\section{Measurements}

Measurements of hysteresis curves were carried out for commercially available samples of $\mathrm{La}(\mathrm{Fe}, \mathrm{Si})_{13}$ alloy using a measurement setup described in [20]. The quasi-static excitation conditions were determined using the classification criterion discussed in detail in [21]. The magnetic circuit was composed of four $\mathrm{LaFe}_{10.8} \mathrm{Co}_{1.1} \mathrm{Si}_{1.1}$ pieces of dimensions $36 \times 18 \times 5 \mathrm{~mm}^{3}$. Our previous paper [22] describes the modeling process which uses a family of hysteresis curves being determined experimentally for different ambient temperatures close to the $T_{\mathrm{C}}(300 \mathrm{~K})$ of the studied material. The excitation frequency for the presented curves was kept at $0.1 \mathrm{~Hz}$ in order to avoid distortion of loops by eddy currents.

\section{Modelling}

As pointed out earlier, hysteresis modelling for MCMs is usually carried out using either the mathematical Preisach approach advanced by Mayergoyz [23] or the macroscopic description proposed by Jiles and Atherton (JA) [24]. The first model is a typical bottom-up approach, where the hysteresis loop is obtained by summing contributions from a number of individual elementary units. The drawback of this approach lies in a difficult interpretation of the Preisach distribution function and its correlation to microstructure of the examined material, yet it should be admitted that some successful attempts were made in the past [25]. On the other hand, 
the JA model is attractive from the engineering perspective, as it has a limited number of parameters and a physical interpretation may be attributed to most of them. However, this description reveals also some drawbacks, related to the assumed form of the basic model equation $[22,26]$. The $T(x)$ phenomenological model based on hyperbolic tangent mapping between the input and output variables may be a valuable and simple alternative to the latter approach. Its foundations are well described, e.g., in the handbook [16]. Apart from the original formulation, this paper considers also a modification which introduces a magnetization dependent function $R(M)=1-\left(M / M_{s}\right)^{2}$, where $M_{s}$ is saturation magnetization. The original $T(x)$ description, which interprets the input variable $x$ as the reduced field strength and the output variable $y$ as the reduced magnetization, was used to compute the irreversible component of the total susceptibility. In other words, the original $T(x)$ model is "inserted" into an additional relationship expressing the total differential susceptibility. A similar approach was applied previously by Kádár to the Preisach description [27, 28], which resulted in a modification of the congruency feature for minor loops. The magnetization dependent function $R(M)$ was interpreted by Basso and Bertotti as a measure of surface of active domain walls, subjected to magnetization process [29]. In this way the interaction of domain walls with microstructure was treated independently of the domain structure itself. The physical processes responsible for losses and reversible susceptibility were not linked to saturation phenomena. This gave rise to the so-called state-independent hypothesis [30]. A symmetrical minor loop is described within the $T(x)$ approach by the following algebraic equation:

$$
M=M_{s} \tanh \left(\frac{H \mp H_{c 0}}{a}\right) \pm b\left(H_{\mathrm{TIP}}, M_{\mathrm{TIP}}\right),
$$

where $H_{c 0}$ is the quasi-static coercive field strength, $M_{s}$ stands for the saturation magnetization, $a$ is a normalization constant which determines the slope of the hysteresis curve, whereas $b$ is introduced in order to match the magnetization values for upper and lower branches at tip points

$$
\begin{aligned}
& b\left(H_{\mathrm{TIP}}, M_{\mathrm{TIP}}\right)=\frac{M_{s}}{2} \tanh \left(\frac{H_{\mathrm{TIP}}+H_{c 0}}{a}\right) \\
& -\frac{M_{s}}{2} \tanh \left(\frac{H_{\mathrm{TIP}}-H_{c 0}}{a}\right) .
\end{aligned}
$$

The quasi-static irreversible differential susceptibility $\mathrm{d} M_{\mathrm{irr}} / \mathrm{d} H$ in the hybrid product- $T(x)$ model is calculated using (1) and (2), whereas the total differential susceptibility is calculated from the dependence

$$
\frac{\mathrm{d} M}{\mathrm{~d} H}=\left(1-\left(\frac{M}{M_{s}}\right)^{2}\right)\left(\beta+\frac{\mathrm{d} M_{\mathrm{irr}}}{\mathrm{d} H}\right) .
$$

The above equation can be integrated analytically, which is an important practical advantage. It can be easily seen that the hybrid model has one degree of freedom more than the original $T(x)$ description, which can blur
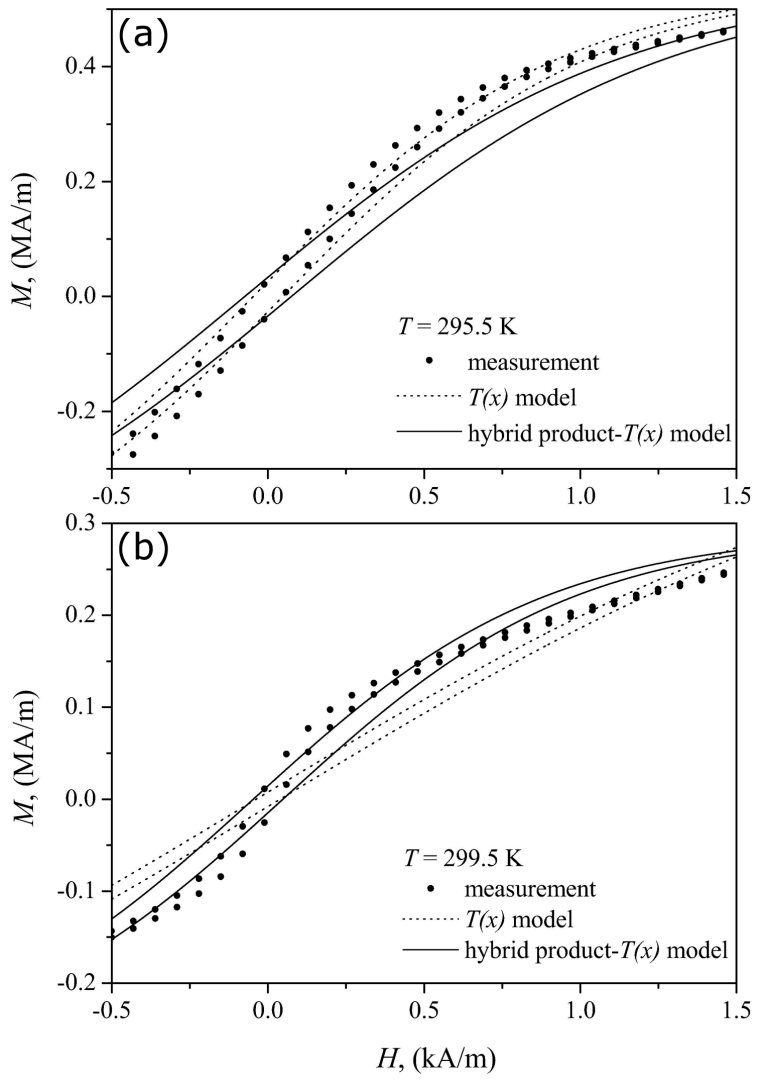

Fig. 1. Measured and modeled hysteresis curves (a) for $295.5 \mathrm{~K}$, and (b) for $299.5 \mathrm{~K}$.

the conclusions drawn from the analysis. An additional term linear with $H$ (the proportionality constant called $c$ ) was added to the relationship (1) in order to compare the performance of both models. The possibility of taking into account the reversible magnetization processes in this way is mentioned in the handbook [16]. During modelling, we have fixed the values of saturation magnetization to the previously determined ones [31] and the values of coercive field strength to their experimental counterparts. The constraint for $c$ was set to $T \geq 0$. We used the solver procedures available in a popular spreadsheet to get the estimated values. We have found out that the original $T(x)$ description with the linear term $c H$ describes quite well the hysteresis curve for the lowest considered temperature (293.5 K). However, upon the temperature increase, the discrepancies between the measured and the modelled curves were more and more evident. Interestingly, the value of parameter $c$ dropped practically to zero for $T \geq 295.5 \mathrm{~K}$.

The hybrid product- $T(x)$ model offered an improved description of magnetization curves closer to the Curie point, which may be inferred from Fig. 1. The calculated values of permeability were less than those obtained from the experiment for the lower temperature values. It can be stated that both considered models yielded qualitatively correct descriptions of the hysteresis loop shape changes versus temperature. 


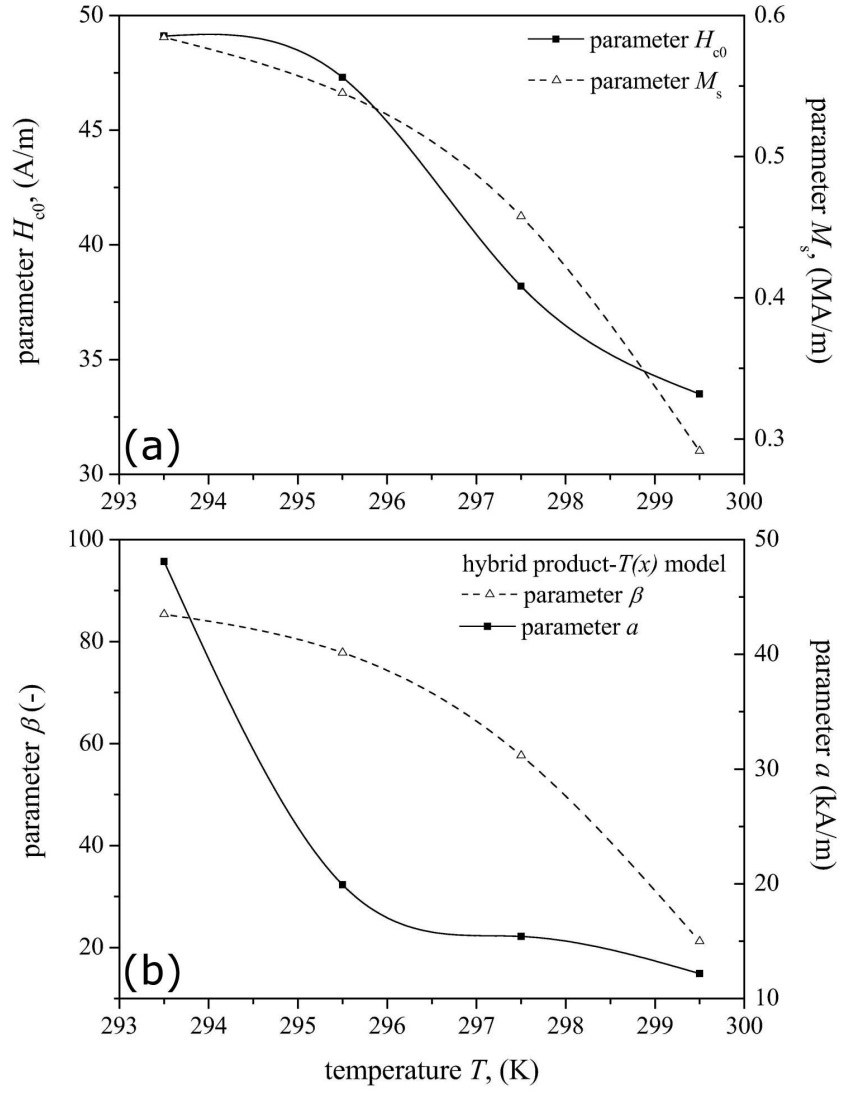

Fig. 2. Trends for the model parameters (a) from previous analyses, and (b) specific to the hybrid model.

Figure 2a depicts the trends for model parameters $H_{c 0}=H_{c 0}(T)$ (the relationship determined experimentally) and $M_{s}=M_{s}(T)$ (determined previously in [31]), while Fig. $2 \mathrm{~b}$ presents the changes in parameters $a=a(T)$ and $\beta=\beta(T)$ appearing in the hybrid description. The parameter $\beta$ is interpreted as the initial reversible susceptibility. It gradually decreases upon the temperature increase, as expected. The parameter $a$, responsible for the shape of the hysteresis curve, drops abruptly within the range of $293.5-295.5 \mathrm{~K}$, its further variations being not so distinct. In order to gain some insight into the possibilities of quantitative description of the hysteresis loop shapes offered by either of the two models, we determined the errors for the remanence points of the curves depicted in Fig. 1. The $T(x)$ model underestimated the value of remanence magnetization by $10.3 \%$ for $295.5 \mathrm{~K}$, whereas the hybrid one overestimated it by $15.2 \%$. Both modeled values were lower than the measured one for $299.5 \mathrm{~K}$. The error was equal either to $56.5 \%$ for the $T(x)$ description, or to $18.6 \%$ for the hybrid model. These values can be compared to those obtained from another description i.e., the GRUCAD model $(93 \%$ and $5.8 \%$, respectively) [22]. It should be remarked that information on quantitative measures and performance of other models is rarely found in literature [20, 32].

\section{Conclusions}

Our paper is focused on changes of hysteresis loop shapes proceeding while the temperature approaches the Curie point. We chose two phenomenological models based on hyperbolic tangent mapping in order to describe this effect. We found out that both approaches were to some extent useful for a quantitative description of the hysteresis curve vanishing at the neighborhood of the PT point. We noticed that the parameter responsible for the reversible contribution suddenly dropped to zero for the $T(x)$ model. Its counterpart $\beta$ used in the hybrid product- $T(x)$ description behaved in a similar way, but the change was less abrupt. Therefore we indicate the latter approach as the preferred one for the description of hysteresis curves of $\mathrm{La}(\mathrm{Fe}, \mathrm{Si})_{13}$ close to the $T_{\mathrm{C}}$. It should be admitted, however, that the original $T(x)$ model describes the $M=M(H)$ dependences very well for lower temperatures, which leads to a conclusion about its usefulness in practical modelling applications. The relatively high level of errors with respect to the remanence value might be related to fixing some of the model parameters to specified values, which limited the number of degrees of freedom for the models.

\section{References}

[1] A. Smith, Eur. Phys. J. H 38, 507 (2013).

[2] V.K. Pecharsky, K.A. Gschneidner Jr., Phys. Rev. Lett. 78, 4494 (1997).

[3] A.M. Tishin, Y.I. Spichkin, The Magnetocaloric Effect and Its Application, Institute of Physics Publ., London 2003.

[4] J. Liu, J.D. Moore, K.P. Skokov, M. Krautz, K. Löwe, A. Barcza, M. Katter, O. Gutfleisch, Scr. Mater. 67, 584 (2012).

[5] V. Franco, J.S. Blázquez, B. Ingale, A. Conde, Ann. Rev. Mater. Res. 42, 305 (2012).

[6] J. Romero Gómez, R. Ferreiro Garcia, A. De Miguel Catoira, M. Romero Gómez, Renew. Sustain. Energy Rev. 17, 74 (2013).

[7] M. Balli, S. Jandl, P. Fournier, A. Kedous-Lebouc, Appl. Phys. Rev. 4, 021305 (2017).

[8] V. Basso, arXiv:1702.08347v1 (2017).

[9] V. Lollobrigida, V. Basso, F. Borgatti, et al., J. Appl. Phys. 115, 203901 (2014).

[10] A. Fujita, S. Fujieda, K. Fukamichi, J. Magn. Magn. Mater. 310, e1006 (2007).

[11] P. Gębara, J. Marcin, I. Škorvanek, J. Electron. Mater. 46(11), 6518 (2017)

[12] P. Gębara, J. Kovač, Mater. Des. 129, 111 (2017).

[13] A. Yan, K.H. Muller, O. Gutfleisch, J. Alloys Comp. 450, 18 (2008).

[14] P. Gębara, P. Pawlik, B. Michalski, J.J. Wysłocki, Acta Phys. Pol. A 127, 576 (2015).

[15] P. Gębara, P. Pawlik, M. Hasiak, J. Magn. Magn. Mater. 422, 61 (2017).

[16] J. Takács, Mathematics of Hysteretic Phenomena, Wiley-VCH, Weinheim 2003. 
[17] K. Chwastek, A.P.S. Baghel, B. Sai Ram, B. Borowik, L. Daniel, S.V. Kulkarni, J. Phys. D Appl. Phys. 51, 145003 (2018).

[18] V. Basso, C.P. Sasso, G. Bertotti, M. LoBue, Physica B 403, 312 (2008).

[19] Y. Melikhov, R.L. Hadimani, A. Raghunathan, J. Appl. Phys. 115, 183902 (2014).

[20] P. Gębara, R. Gozdur, K. Chwastek, in: Proc. Conf. 2018 Progress in Applied Electrical Engineering, Koscielisko (Poland), 2018, p. 8441091.

[21] R. Gozdur, A. Majocha, Przegl. Elektrotechn. 1, 134 (2007).

[22] R. Gozdur, P. Gębara, K. Chwastek, Open Phys. 16, 266 (2018).

[23] I.D. Mayergoyz, Mathematical Models of Hysteresis and Their Applications, 2nd ed., Academic Press, 2003.

[24] D.C. Jiles, D.L. Atherton, J. Magn. Magn. Mater 61, 48 (1986).
[25] V. Basso, G. Bertotti, A. Infortuna, M. Pasquale, IEEE Trans. Magn. 314000 (1995).

[26] S.E. Zirka, Yu.I. Moroz, R.G. Harrison, K. Chwastek, J. Appl. Phys. 112, 043916 (2012).

[27] G. Kádár, Phys. Scr. T25, 161 (1989).

[28] G. Kádár, Physica B 275, 40 (2000).

[29] V. Basso, G. Bertotti, IEEE Trans. Magn. 32, 4210 (1996).

[30] M. Carpentieri, B. Azzerboni, G. Finocchio, F. La Foresta, J. Magn. Magn Mater. 280, 158 (2004).

[31] R. Gozdur, K. Chwastek, M. Najgebauer, M. Lebioda, Ł. Bernacki, A. Wodzyński, Acta Phys. Pol. A 131, 801 (2017).

[32] V. Basso, M. Kuepferling, C.P. Sasso, M. LoBue, IEEE Trans. Magn. 44, 3177 (2008). 